Introduction: Epithelial-mesenchymal transition (EMT) is important in the metastasis of tumours and is triggered by several key growth factors, including platelet-derived growth factor-B (PDGF-B). But, whether PDGF-B signalling promotes $E M T$ in gastric carcinoma cells is still unknown.

Material and methods: We established 2 gastric carcinoma cell lines (MKN28 and MKN45) to stably overexpress PDGF-B by lentiviral vectors, and expression of E-cadherin, N-cadherin, and ERK-1 were detected by western blot assay. Then, PDGF-B overexpression and normal MKN28 and MKN45 cells were cocultured with PDGFR- $\beta$ positive fibroblast (hs738) and MAPK inhibitors were added; also, the expressions of ERK-1, E-cadherin, and $\mathrm{N}$-cadherin were detected by western blot assay.

Results: After being cocultured with hs738 cells, expressions of ERK-1 and $\mathrm{N}$-cadherin protein in PDGF-B overexpression MKN28 and MKN45 cells were much higher than normal MKN28 and MKN45 cells ( $p<0.05)$, and those could be decreased by MAPK inhibitor. Also, expressions of E-cadherin protein in PDGF-B overexpression MKN28 and MKN45 cells were much lower than normal MKN28 and MKN45 cells $(p<0.05)$, and they could be increased by MAPK inhibitor. Conclusions: Our data indicate that PDGF-B signalling can induce EMT in gastric carcinoma cells. Thr tumour microenvironment is imperative in the process of PDGF-B signalling inducing EMT in gastric carcinoma cells. Also, activation of MAPK/ERK pathway, which is a downstream pathway of PDGF-B signalling, might participate in this process.

Key words: PDGF-B signalling, EMT, E-cadherin, N-cadherin, MAPK/ERK pathway.

Contemp Oncol (Pozn) 2021; 25 (1): 1-6 DOI:https://doi.org/10.5114/wo.2021.103938

\section{Platelet-derived growth factor-B signalling might promote epithelial-mesenchymal transition in gastric carcinoma cells through activation of the MAPK/ERK pathway}

\author{
Jiangyan Yin ${ }^{1}$, Yi Guo ${ }^{2}$, Zhongfu Li
}

${ }^{1}$ Department of Ultrasound, The First Affiliated Hospital of Chongqing Medical University, Chongqing, China

2Department of General Surgery, Chongqing University Central Hospital (Chongqing Emergency Medical Center), Chongqing, China

\section{Introduction}

In the past few decades, mortality caused by gastric cancer has decreased [1], but gastric cancer is still the second leading cause of cancer mortality in the world [2]. Because metastasis is the most important cause of death, many efforts have been focused on better understanding of its mechanisms. Emerging evidence suggests that epithelial-mesenchymal transition (EMT) is quite important in the metastasis of various kinds of solid tumours $[3,4]$, including gastric carcinomas [5]. However, the mechanism that leads tumours to induce EMT is poorly understood.

Epithelial-mesenchymal transition is a coordinated molecular and cellular change defined as a reduction in cell-cell adhesion, apical-basolateral polarity, and epithelial markers, as well as an acquisition of motility, spindle-cell shape, and mesenchymal markers [6]. This process involves a disassembly of cell-cell junctions, including downregulation and relocation of E-cadherin as well as upregulation and relocation of N-cadherin [7]; hence, both $\mathrm{E}$-cadherin and $\mathrm{N}$-cadherin are important molecular markers of EMT. Also, the processes of EMT can be triggered by various growth factors, such as platelet-derived growth factor-B (PDGF-B) [8-10]. Recently, Michiyo Kodama et al. [11] showed that PDGF-B secreted by tumour cells and PDGFR- $\beta$ expressed by stromal cells are associated with lymphatic metastasis in gastric carcinoma, and they inferred that the tumour microenvironment might play quite an important role in PDGF-B-induced tumour metastasis. However, the mechanism by which PDGF-B signalling promotes metastasis in human gastric carcinoma is still unknown. Also, MAPK/ERK signalling, an active tumour metastasis-relating pathway, is widely reported to be activated by many kinds of growth factors to promote EMT of tumour cells [12, 13]. So, we conjectured that PDGF-B signalling might induce EMT to promote metastasis via activation of MAPK/ERK signalling in human gastric carcinoma.

In our study, we established two gastric carcinoma cell lines, MKN28 and MKN45, to stably overexpress PDGF-B by lentiviral vectors, and detected their expressions of E-cadherin, N-cadherin, and ERK-1. Then, PDGF-B overexpression MKN28 and MKN45 cells were cocultured with PDGFR- $\beta$-positive fibroblast (hs738) cells to construct the activation of PDGF-B signalling. After that the MAPK inhibitor was added. Finally, we detected their expressions of E-cadherin, N-cadherin, and ERK-1 to ascertain whether PDGF-B signalling promoted EMT and its mechanism. 


\section{Material and methods}

\section{MAPK inhibitor}

MAPK inhibitor VIII, Isozyme-Selective, ERKi-1/2 (Santa Cruz Biotechnology Inc., USA) was used in this study at a concentration of $0.1 \mu \mathrm{g} / \mathrm{ml}$.

\section{Cell culture}

MKN28 and MKN45 human gastric carcinoma cell lines and fibroblast (hs738) were provided by the Cell Bank of the Chinese Academy of Sciences, Shanghai, China. Cells were cultured in Roswell Park Memorial Institute 1640 (RPMI1640) media supplemented with 10\% foetal bovine serum (FBS).

\section{Lentiviral vector constructs and preparation}

A lentiviral-delivered PDGF-B vector was constructed and prepared by Chongqing Western Technology Inc. (Chongqing, China) as described by Lois et al. [14] and Xia et al. [15]. Briefly, primers were designed according to the PDGF-B sequence (Genbank Accession Number NM_002608.2). The following primer sequences were used: PDGF-B-F, 5'- ATGAATCGCTGCTGGGCGCTC-3'; PDGF-B-R, 5'-CTAGGCTCCAAGGGTCTCCTTC-3'. The target gene was obtained by polymerase chain reaction $(P C R)$ and was inserted into the pUC57 vector. Then, both the pLenO-DCEand PUC57-PDGF-Bwere enzyme digested byECoR I and Not I, respectively. After ligation, the pLenO-DCE-PDGF-B vector was constructed. After sequencing, the pLenODCE-PDGF-B vector was transfected into 293T cells and lentiviral-delivered PDGF-B vector was prepared.

\section{Cell transfection}

Briefly, $1 \times 10^{5}$ MKN28 and MKN45 cells were seeded in each well of a 6 -well plate in $500 \mu$ l of complete media at $37^{\circ} \mathrm{C}$ in a $5 \% \mathrm{CO}_{2}$ incubator for $24 \mathrm{~h}$, and then transduced by lentiviral vectors at a multiplicity of infection of $10: 1$ [16]. Transduction was carried out in the presence of Polybrene $(8 \mu \mathrm{g} / \mathrm{ml})$. After washing 3 times with PBS, $1 \mathrm{ml}$ of RPMI1640 was added to each well. Cells were seeded at $37^{\circ} \mathrm{C}$ in a $5 \%$ $\mathrm{CO}_{2}$ incubator for $48 \mathrm{~h}$. Fluorescence microscopy was used to observe the transduction. $400 \mu \mathrm{g} / \mathrm{ml}$ of green fluorescent proteins was used for screening. Transduced cells were passaged and seeded for further experiments.

\section{Cell coculture}

Stratified co-culture technique was used for cell coculture [17]. Normal gastric carcinoma cells (MKN28 and MKN45) and fibroblast (hs738) cells, PDGF-B overexpression gastric carcinoma cells (MKN28 and MKN45), and fibroblast (hs738) cells were cocultured $\left(1.5 \times 10^{5}\right.$ of each cell type) in 6-well Transwells (Corning Inc., Corning, NY) for $72 \mathrm{~h}$ [17]. The fibroblast (hs738) cells were plated in RPMI1640 (10\% FBS) at $37^{\circ} \mathrm{C}$ on the underside of the Transwells. After $90 \mathrm{~min}$, the Transwells were reinserted into the 6-well plate and normal gastric carcinoma cells (MKN28 and MKN45) or PDGF-B overexpression gastric carcinoma cells (MKN28 and MKN45) were plated in the upper chamber of the Transwell. After being cocultured for $72 \mathrm{~h}$, normal gastric carcinoma cells (MKN28 and MKN45) or
PDGF-B overexpression gastric carcinoma cells (MKN28 and MKN45) were gathered.

\section{Western blot analysis}

Cells were lysed on ice in RIPA buffer $(50 \mathrm{mM}$ Tris- $\mathrm{HCl}$, $150 \mathrm{mM} \mathrm{NaCl}, 1 \%$ NP-40, 0.1\% SDS, 0.5\% sodium deoxycholate, $2 \mathrm{mM}$ sodium fluoride, $2 \mathrm{mM} \mathrm{Na}_{3} \mathrm{VO}_{2}, 1 \mathrm{mM}$ EDTA, and $1 \mathrm{mM}$ EGTA). Total protein extracts were analysed by western blotting, as described previously [18]. Proteins $(20 \mu \mathrm{g})$ were separated by SDS-PAGE gels (Invitrogen) and transferred to PVDF membranes. The membranes were blotted for $1 \mathrm{~h}$ with 5\% milk. Membranes were incubated with primary antibodies (1: 500 dilution) against E-cadherin, N-cadherin, or ERK-1 (Santa Cruz Biotechnology, Inc., USA) at $4^{\circ} \mathrm{C}$ overnight. After incubation with horseradish peroxidase-conjugated secondary antibody (1 : 1000 dilution) for $3 \mathrm{~h}$ at $37^{\circ} \mathrm{C}$, signals were detected by $\mathrm{ECL}$ chemiluminescence for $5 \mathrm{~min}$. The films were analysed by densitometry with image software.

\section{Statistics analysis}

Data were expressed as mean \pm SE and were statistically evaluated by $t$-test. The difference was considered significant if $p<0.05$.

\section{Results}

Construction and evaluation of platelet-derived growth factor-B overexpression MKN28 and MKN45 gastric carcinoma cells

To check the overexpression of PDGF-B in our stably transfected cells, we checked the immunofluorescence and expression of PDGF-B protein in our transfected MKN28 and MKN45 gastric carcinoma cells, which were transduced with lentiviral constructs to overexpress PDGF-B. As illustrated in Fig. 1, our stably transduced cells showed significant green fluorescence. Also, the protein levels of PDGF-B increased significantly in transfected cells $(p<0.05)$ (Fig. 2).

Expression of E-cadherin, N-cadherin, and ERK-1 in platelet-derived growth factor-B overexpression and normal MKN28 and MKN45 gastric carcinoma cells

We determined the expressions of E-cadherin, N-cadherin, and ERK-1 protein in PDGF-B overexpression MKN28 and MKN45 cells and normal MKN28 and MKN45 cells by western blot analysis. As illustrated in Fig. 3, expressions of E-cadherin, N-cadherin, and ERK-1 protein were no different between PDGF-B overexpression MKN28 and MKN45 cells and normal MKN28 and MKN45 and cells $(p>0.05)$ (Fig. 3).

Expression of E-cadherin, N-cadherin, and ERK-1 in platelet-derived growth factor-B overexpression and normal MKN28 and MKN45 gastric carcinoma cells after coculture

For activation of PDGF-B signalling, both normal gastric carcinoma cells (MKN28 and MKN45) and PDGF-B overexpression gastric carcinoma cells (MKN28 and MKN45) were cocultured with fibroblast (hs738) cells. Then, ex- 
MKN45

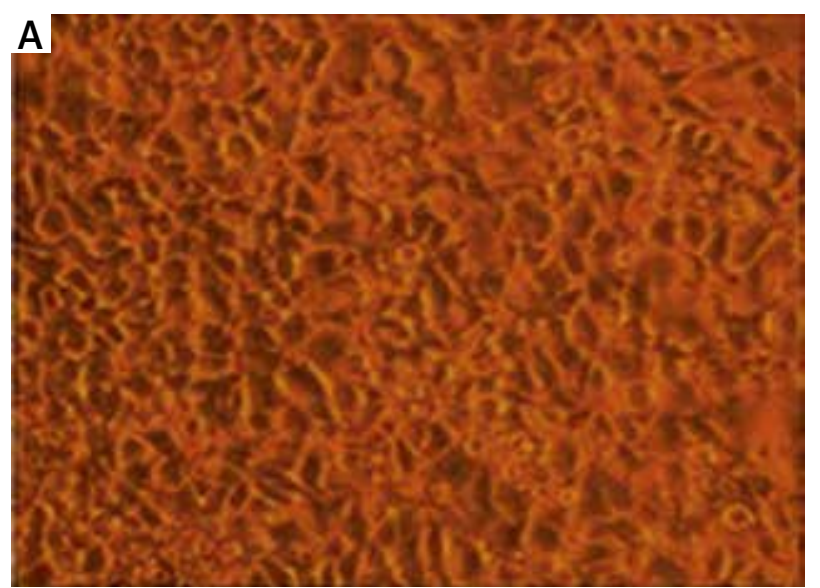

C

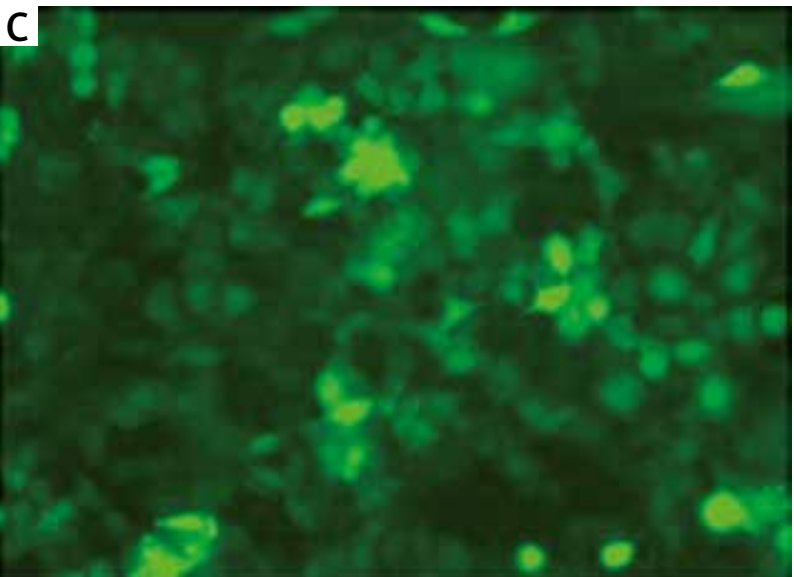

MKN28
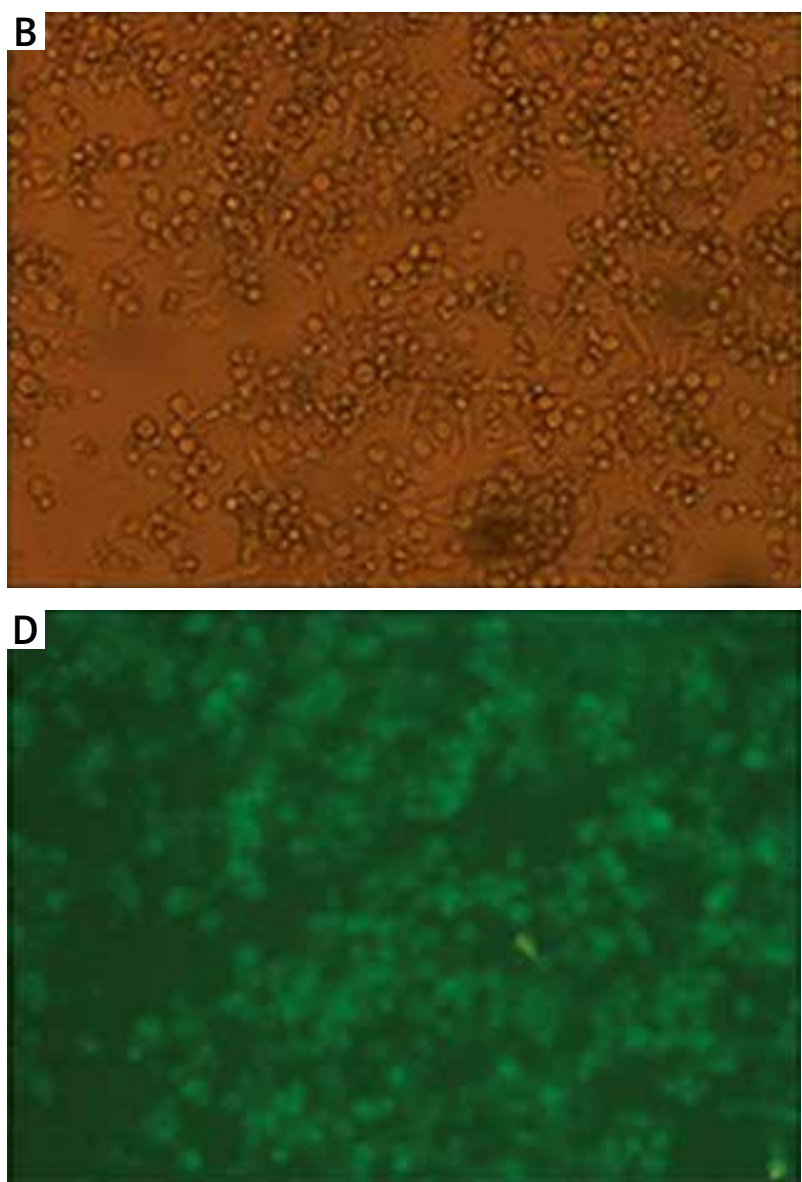

Fig. 1. Immunofluorescence of MKN45 and MKN28 gastric carcinoma cells after transfection of platelet-derived growth factor-B lentiviral vector $(\times 200)$

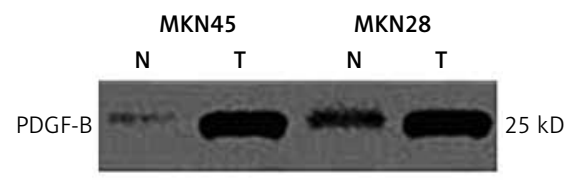

GAPDH

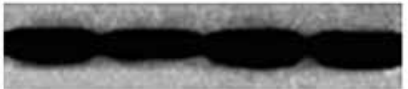

$36 \mathrm{kD}$

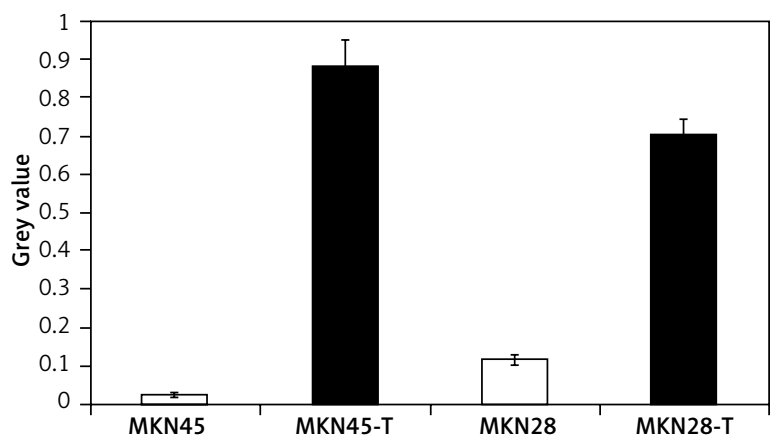

$N$ - normal cells, $T$ - transduced cells

Fig. 2. Platelet-derived growth factor-B protein high expression in transfected MKN45 and MKN28 gastric carcinoma cells
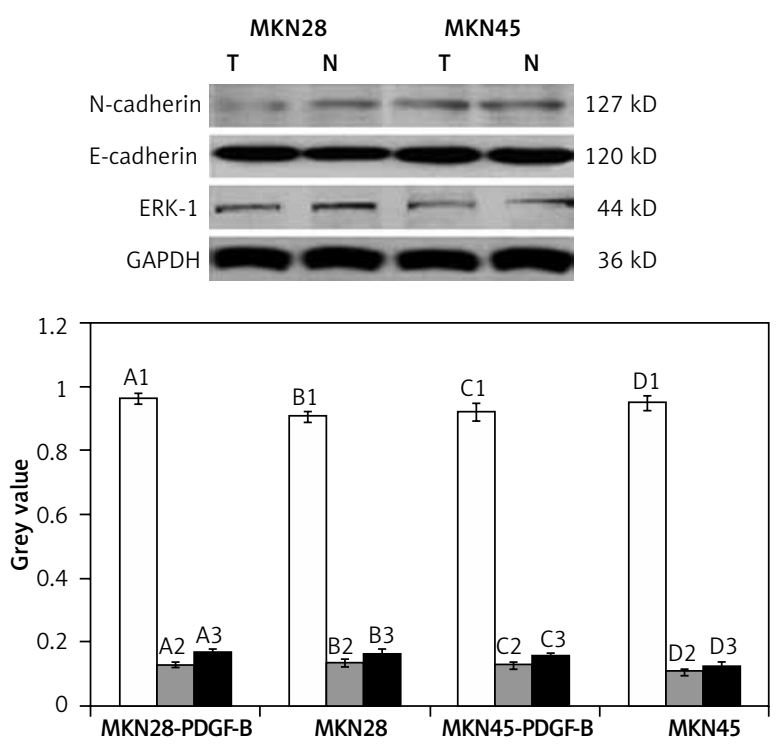

$N$ - normal cells, $T$-transduced cells; A1 compared with B1 and C1 with compared D1 $p>0.05$, A2 compared with B2 and C2 compared with D2, $p>0.05, A 3$ compared with B3 and C3 compared with D3, $p>0.05$

Fig. 3. Overexpression of platelet-derived growth factor-B might not change the expression of ERK-1, E-cadherin, and $\mathrm{N}$-cadherin protein in MKN28 and MKN45 gastric carcinoma cells 

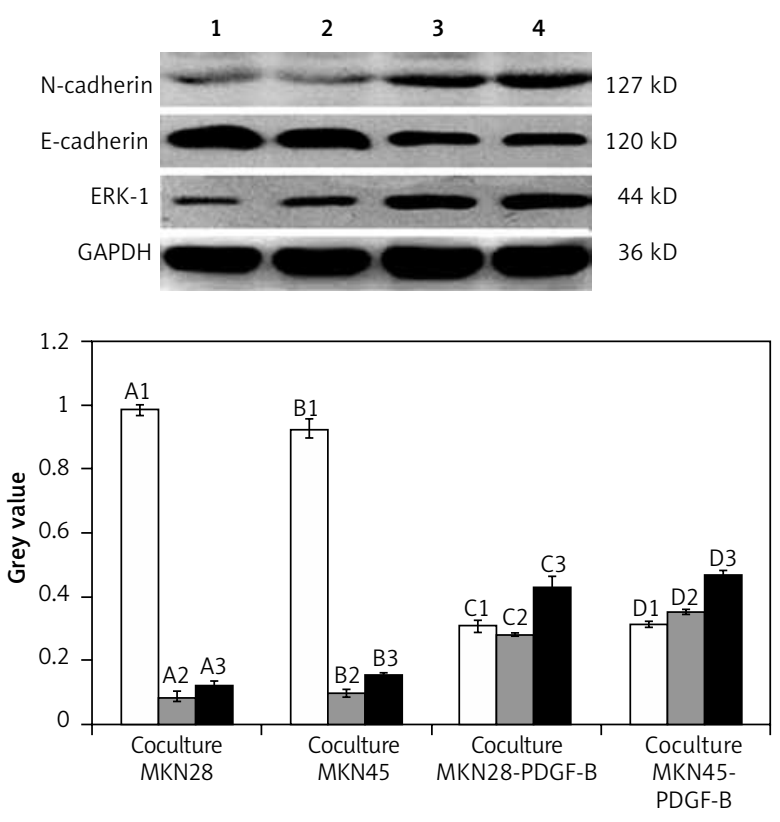

$\square$ E-cadherin $\square$ N-cadherin $\square$ ERK-1

1 - coculture MKN28, 2 - coculture MKN45, 3 - coculture MKN28-PDGF-B, 4 - coculture MKN45-PDGF-B; A1 compared with C1, A2 compared with C2 and A3 compared with C3, $p<0.05, B 1$ compared with D1, B2 compared with D2 and $B 3$ compared with D3, $p<0.05$

Fig. 4. Expression of ERK-1 and N-cadherin increased, and expression of E-cadherin decreased in cocultured platelet-derived growth factor-B (PDGF-B) overexpression MKN28 and MKN45 gastric carcinoma cells

pressions of E-cadherin, N-cadherin, and ERK-1 protein in PDGF-B overexpression and normal MKN28 and MKN45 cells were detected by western blot analysis. As illustrated in Fig. 4, expression of N-cadherin and ERK-1 protein increased significantly, and expression of E-cadherin decreased significantly $(p<0.05)$ (Fig. 4).

Expression of E-cadherin, $\mathrm{N}$-cadherin, and ERK-1 in cocultured platelet-derived growth factor-B overexpression MKN28 and MKN45 gastric carcinoma cells after adding MAPK inhibitor

For further detection of the role of the MAPK/ERK pathway in EMT induced by activation of PDGF-B signalling, MAPK inhibitors were added into cocultured PDGF-B overexpression gastric carcinoma cells (MKN28 and MKN45). Then, expressions of E-cadherin, N-cadherin, and ERK-1 protein in cocultured PDGF-B overexpression MKN28 and MKN45 cells were detected by western blot analysis. As illustrated in Fig. 5, expressions of N-cadherin and ERK-1 protein were decreased significantly by MAPK inhibitor, and expressions of E-cadherin were increased significantly by MAPK inhibitor $(p<0.05)$ (Fig. 5).

\section{Discussion}

Metastasis is a common clinical finding in many human cancers, and it is the primary cause of death for most cancer patients $[19,20]$. For many years, scientists have tried to understand the mechanisms of tumour metasta-
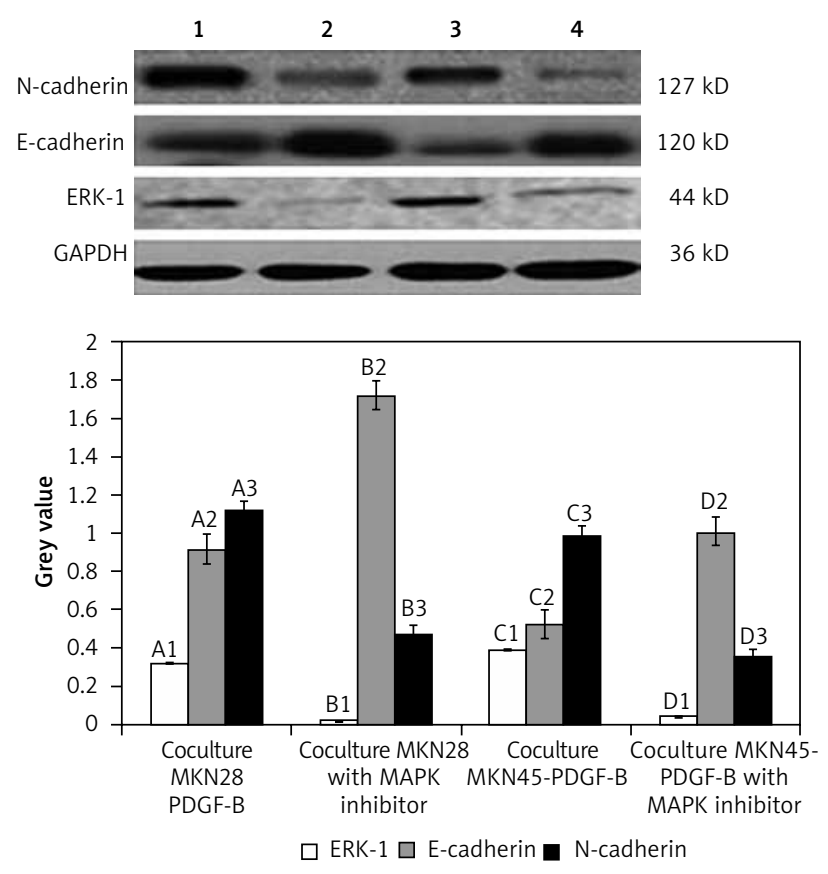

1 - coculture MKN28-PDGF-B, 2 - coculture MKN28-PDGF-B with MAPK inhibitor, 3 - coculture MKN45-PDGF-B, 4- coculture MKN45-PDGF- $B$ with MAPK inhibitor; $A 1$ compared with $B 1, A 2$ compared with $B 2$ and $A 3$ compared with $B 3, p<0.05, C 1$ compared with D1, C2 compared with D2 and C3 compared with $D 3, p<0.05$

Fig. 5. MAPK inhibitor could increase the expression of E-cadherin and decrease the expression of N-cadherin and ERK-1 in cocultured platelet-derived growth factor-B (PDGF-B) overexpression MKN28 and MKN45 gastric carcinoma cells

sis, but the results are unsatisfactory. Although the exact mechanisms of metastasis are still unknown, it is widely accepted that EMT is of vital importance in the process of tumour metastasis [5-7]. PDGF-B signalling is one of the important growth factors that were demonstrated to be concerned with EMT [10, 21]. Also, PDGF-B signalling was highly expressed in gastric cancer cells, and its specific receptor PDGFR- $\beta$ was highly expressed in tumour stromal cells of gastric cancer $[22,23]$. Hence, we inferred that activation of PDGF-B signalling might induce EMT to promote tumour metastasis in gastric cancer. EMT is a complex molecular and cellular process and is regulated by varied of biology factors [24]. EMT is a coordinated molecular and cellular change defined as a reduction in cell-cell adhesion, apical-basolateral polarity, and epithelial markers, as well as an acquisition of motility, spindle-cell shape, and mesenchymal markers [6]. Many EMT-associated markers, such as epithelial specific markers (i.e. E-cadherin and cytokeratin) [25] and mesenchymal specific markers (i.e. N-cadherin and vimentin) [26, 27], were used for the detection of EMT. In our study, we tried to verify that activation of PDGF-B signalling might promote EMT in gastric cancer cells and tried to explore its mechanisms.

In our study, we found that PDGF-B overexpression MKN28 and MKN45 gastric carcinoma cells follow EMT just after being cocultured with cancer-associated PDGFR- $\beta$-positive fibroblast. The result showed the indispensable role of cancer-associated PDGFR- $\beta$-positive fibroblast 
in the EMT of gastric carcinoma cells induced by activation of PDGF-B signalling. Cancer-associated fibroblasts, the same as extracellular matrix (ECM), myofibroblasts, immune cells, and soluble factors, were an important component of tumour microenvironment $[28,29]$ and were widely involved in the process of EMT in many cancer cells [30-32]. Also, many studies showed that interaction among cancer cells in the tumour microenvironment can induce EMT by many mediators such as growth factors, cytokines, and ECM proteins [33, 34]. Hence, we inferred that PDGF-B, an important growth factor, could induce EMT in gastric carcinoma cells through interaction with the tumour microenvironment. The potential mechanisms might be that activation of PDGF-B signalling could induce the variations of tumour stromal cells so as to change the tumour microenvironment, such as hypoxia, acid environment, hypermetabolism, and so on [35, 36]. However, the exact mechanism(s) of how the tumour microenvironment regulates activation of PDGF-B signalling and promotes EMT is/are still not yet determined.

Also, for further exploration of the potential mechanism of how activation of PDGF-B signalling promotes EMT, MAPK/ERK signalling, a prominent tumour metastasis-related downstream pathway was detected. MAPK/ERK signalling [37] could be activated by many kinds of growth factors to promote EMT of tumour cells [38-40]. Moreover, many studies have documented that PDGF-B plays a significant role in gastric carcinoma metastasis [11, 22]. Hence, we sought to investigate whether the effects of PDGF-B signalling activation on metastasis of gastric carcinoma was mediated through activation of MAPK/ERK signalling. Significantly, activation of PDGF-B signalling increased the expression of ERK-1 and N-cadherin protein and decreased the expression of E-cadherin protein in MKN28 and MKN45 gastric carcinoma cells. Also, when PDGF-B signalling activation MKN28 and MKN45 gastric carcinoma cells were treated with MAPK inhibitor, the expression of $\mathrm{N}$-cadherin protein was decreased and the expression of $\mathrm{E}$-cadherin protein was increased. We could infer that activation of PDGF-B signalling induces EMT of gastric carcinoma cells at least partially through the activation of MAPK/ERK signalling. It is well known that MAPK inhibitor might inhibit the activation of ERK-1, but it was quite strange that when MAPK inhibitor was added, the expression of ERK-1 protein was decreased. We speculated that MAPK inhibitor might inhibit the activity of PDGF-B signalling through some mechanisms [41]. First, downstream pathways of MAPK/ERK signalling might be inhibited, and negative feedbacks were transferred to PDGF-B signalling. Then, activation of PDGF-B signalling was decreased [42]. However, the exact mechanism(s) of how PDGF-B signalling regulates the MAPK/ERK signalling is/are still not yet determined.

\section{Conclusions}

We found that PDGF-B signalling can induce EMT in gastric carcinoma cells. The tumour microenvironment is imperative in the process of PDGF-B signalling inducing EMT in gastric carcinoma cells. Also, activation of the MAPK/ERK pathway, which is a downstream pathway of
PDGF-B signalling, might participate in this process. Thus, blockage of PDGF-B signalling pathway may be a reasonable approach to the treatment of gastric carcinoma.

\section{Acknowledgments}

This article was funded by Chongqing Science and Technology Bureau (NO: cstc2018jcyj-AX0740 and cstc2020 jcyjmsxm3181).

The authors declare no conflict of interest.

\section{References}

1. Siegel RL, Miller KD, Jemal A. Cancer Statistics, 2017. CA Cancer J Clin 2017; 67: 7-30.

2. Torre LA, Bray F, Siegel RL, et al. Global cancer statistics, 2012. CA Cancer J Clin 2015; 65: 87-108.

3. Lamouille S, Xu J, Derynck R. Molecular mechanisms of epithelialmesenchymal transition. Nat Rev Mol Cell Biol 2014; 15: 178-196.

4. Yang J, Weinberg RA. Epithelial-mesenchymal transition: at the crossroads of development and tumor metastasis. Dev Cell 2008; 14: 818-829.

5. Kim MA, Lee HS, Lee HE, et al. Prognostic importance of epithelialmesenchymal transition-related protein expression in gastric carcinoma. Histopathology 2009; 54: 442-451.

6. Takanori T, Soichiro I, Guo-fu H. Epithelial-mesenchymal transition and cell cooperativity in metastasis. Cancer Res 2009; 69: 7135 7139.

7. Qi X, Zhang L, Lu X. New insights into the epithelial-to-mesenchymal transition in cancer. Trends Pharmacol Sci 2016; 37: 246-248.

8. Wu X, Tao P, Zhou Q, et al. IL-6 secreted by cancer-associated fibroblasts promotes epithelial-mesenchymal transition and metastasis of gastric cancer via JAK2/STAT3 signaling pathway. Oncotarget 2017; 8: 20741-20750.

9. Shareen I, Shumin Z, Adel D, et al. PDGF upregulates Mcl-1 through activation of $\beta$-catenin and HIF-1 $\alpha$-dependent signaling in human prostate cancer cells. PLoS ONE 2012; 7: e30764-30771.

10. Patel P, West-Mays J, Kolb M, et al. Platelet derived growth factor $B$ and epithelial mesenchymal transition of peritoneal mesothelial cells. Matrix Biol 2010; 29: 97-106.

11. Kodama M, Kitadai Y, Sumida T, et al. Expression of plateletderived growth factor (PDGF)-B and PDGF-receptor $b$ is associated with lymphatic metastasis in human gastric carcinoma. Cancer Sci 2010; 101: 1984-1989.

12. Chiara G, Simona D, Chiara R, et al. The A2B adenosine receptor modulates the epithelial-mesenchymal transition through the balance of CAMP/PKA and MAPK/ERK pathway activation in human epithelial lung cells. Front Pharmacol 2018; 9: 54-61.

13. Zhou Q, Chen J, Feng J, et al. SOSTDC1 inhibits follicular thyroid cancer cell proliferation, migration, and EMT via suppressing PI3K/ Akt and MAPK/Erk signaling pathways. Mol Cell Biochem 2017; 435: 87-95.

14. Lois C, Hong EJ, Pease S, Brown EJ, Baltimore D. Germline transmission and tissue-specific expression of transgenes delivered by lentiviral vectors. Science 2002; 295: 868-872.

15. Hager S, Frame FM, Collins AT, et al. An internal polyadenylation signal substantially increases expression levels of lentivirus-delivered transgenes but has the potential to reduce viral titer in a promoter-dependent manner. Hum Gene Ther 2008; 19: 840-850.

16. Huang YM, Zheng Y, Li JG, et al. Lentivirus-mediated RNA interference targeting FAMLF-1 inhibits cell growth and enhances cell differentiation of acute myeloid leukemia partially differentiated cells via inhibition of AKT and c-MYC. Oncotarget 2017; 8: 101372101382.

17. Ye X, Abou-Rayyah Y, Bischoff J, et al. Altered ratios of pro- and anti-angiogenic VEGF-A variants and pericyte expression of DLL4 
disrupt vascular maturation in infantile haemangioma. J Pathol 2016; 239: 139-151.

18. Kong D, Li Y, Wang Z, Banerjee S, Sarkar FH. Inhibition of angio genesis and invasionby 3,3'-diindolylmethane is mediated by the nuclear factor-kappaB downstream target genes MMP-9 and UPA that regulated bioavailability of vascular endothelial growth factor in prostate cancer. Cancer Res 2007; 67: 3310-3319.

19. Steeg PS. Tumor metastasis: mechanistic insights and clinical challenge. Nat Med 2006; 12: 895-904.

20. Dang O, Liu J, Li J, et al. Podoplanin: a novel regulator of tumor invasion and metastasis. Med Oncol 2014; 31: 24-29.

21. Yang LQ, Lin CR, Liu ZR. P68 RNA helicase mediates PDGF-induced epithelial mesenchymal transition by displacing axin from $\beta$-catenin. Cell 2006; 126: 139-155.

22. Suzuki S, Dobashi Y, Hatakeyama Y, et al. Clinicopathological significance of platelet-derived growth factor (PDGF)-B and vascular endothelial growth factor-A expression, PDGF receptor-b phosphorylation, and microvessel density in gastric cancer. Cancer 2010; 10: 659-669.

23. Guo Y, Yin J, Zha L, et al. Clinicopathological significance of platelet-derived growth factor $\mathrm{B}$, platelet-derived growth factor receptor- $\beta$, and $\mathrm{E}$-cadherin expression in gastric carcinoma. Contemp Oncol (Pozn) 2013; 17: 150-155.

24. Voulgari A, Pintzas A. Epithelial-mesenchymal transition in cancer metastasis: mechanisms, markers and strategies to overcome drug resistance in the clinic. Biochim Biophys Acta 2009; 1796: 75-90.

25. Veaceslav F, Lucian R, Amalia RB, et al. Differential expression of e-cadherin in primary breast cancer and corresponding lymph node metastases. Anticancer Res 2015; 35: 759-765.

26. Dauphin M, Barbe C, Lemaire S, et al. Vimentin expression predicts the occurrence of metastases in non-small cell lung carcinomas. Lung Cancer 2013; 81: 117-122.

27. Gagliano N, Celesti G, Tacchini L, et al. Epithelial-to-mesenchyma transition in pancreatic ductal adenocarcinoma: Characterization in a 3D-cell culture model. World J Gastroenterol 2016; 22: 4466 4483.

28. Lorusso G, Rüegg C. The tumor microenvironment and its contribution to tumor evolution toward metastasis. Histochem Cell Biol 2008; 130: 1091-1103.

29. Tao L, Huang G, Song $H$, et al. Cancer associated fibroblasts: an essential role in the tumor microenvironment (review). Onco Lett 2017; 14: 2611-2620.

30. Yang L, Lin PC. Mechanisms that drive inflammatory tumor microenvironment, tumor heterogeneity, and metastatic progression. Semin Cancer Biol 2017; 47: 185-195.

31. Giannoni E, Bianchini F, Masieri L, et al. Reciprocal activation of prostate cancer cells and cancer-associated fibroblasts stimulates epithelial-mesenchymal transition and cancer stemness. Cancer Res 2010; 70: 6945-6956

32. Vered M, Dayan D, Yahalom R, et al. Cancer-associated fibroblasts and epithelial-mesenchymal transition in metastatic oral tongue squamous cell carcinoma. Int J Cancer 2010; 126: 1356-1362.

33. Liu Y, Kong D, Wu H, et al. Interplay of retinal determination gene network with TGF- $\beta$ signaling pathway in epithelial-mesenchymal transition. Stem Cell Investigation 2015; 2: 1-7.

34. Masaaki I, Koshi M, Takehiko Y, et al. Epithelial-mesenchymal transition in cancer development and its clinical significance. Cancer Sci 2010; 101: 293-299.

35. Barber A, Rynda A, Sentman CL. Tumor microenvironment. J Immu nol 2014; 193: 1513

36. Tse JC, Kalluri R. Mechanisms of metastasis: epithelial-to-mesenchymal transition and contribution of tumor microenvironment. J Cell Biochem 2010; 101: 816-829.

37. Santarpia L, Lippman SM, El-Naggar AK. Targeting the MAPK-RAS RAF signaling pathway in cancer therapy. Expert Opin Ther Targets 2012; 16: 103-119.

38. Guo H, Zhang XY, Peng J, et al. RUVBL1, a novel C-RAF-binding protein, activates the RAF/MEK/ERK pathway to promote lung cancer tumorigenesis. Biochem Biophys Res Commun 2018; 498: 932-939.

39. Wong KK. Recent developments in anti-cancer agents targeting the Ras/Raf/ MEK/ERK pathway. Recent Pat Anticancer Drug Discov 2009; $4: 28-35$
40. Chen XF, Zhang HJ, Wang HB, et al. Transforming growth factor- $\beta 1$ induces epithelial-to-mesenchymal transition in human lung cancer cells via PI3K/Akt and MEK/Erk1/2 signaling pathways. Mol Biol Rep 2012; 39: 3549-3556.

41. Rafty LA. Novel negative regulatory element in the platelet-derived growth factor B chain promoter that mediates ERK-dependent transcriptional repression. J Biol Chem 2000; 275: 11478-11483.

42. Dixon BS, Evanoff D, Fang WB, et al. Bradykinin B1 receptor blocks PDGF-induced mitogenesis by prolonging ERK activation and increasing p27Kip1.[J]. Am J Physiol Cell Physiol 2002; 283: C193.

\section{Address for correspondence}

\section{PhD Yi Guo}

Department of General Surgery

Chongqing University Central Hospital

(Chongqing Emergency Medical Center)

Jiankang Road $1^{\text {st }}$ of Yuzhong district

Chongqing, China, 400014

Phone: 0086-023-63692100

E-mail: guoyi429@163.com

Submitted: 11.09 .2020

Accepted: 30.11 .2020 